\title{
A Highly Sensitive Potentiometric Amphetamine Microsensor Based on All-Solid-State Membrane Using a New Ion-Par Complex, $\left[3,3^{\prime}-\mathrm{Co}\left(1,2-\text { closo- } \mathrm{C}_{2} \mathrm{~B}_{9} \mathrm{H}_{11}\right)_{2}\right]^{-} \mathrm{C}_{9} \mathrm{H}_{13} \mathrm{NH}^{++}$
}

\author{
J. Gallardo-Gonzalez 1,* , A. Baraket ${ }^{1}$, S. Boudjaoui ${ }^{1}$, Y. Clément ${ }^{1}$, A. Alcácer ${ }^{2}$, A. Streklas ${ }^{2}$, \\ F. Teixidor ${ }^{3}$, N. Zine ${ }^{1}$, J. Bausells ${ }^{2}$ and A. Errachid ${ }^{1}$ \\ 1 Institut des Sciences Analytiques, Université de Lyon, UMR 5280, CNRS, Université de Lyon 1, ENS Lyon-5, \\ 5 rue de la Doua, 69100 Villeurbanne, France; Abdoullatif.BARAKET@isa-lyon.fr (A.B.); \\ selim.boudjaoui@isa-lyon.fr (S.B.); yohann.clement@univ-lyon1.fr (Y.C.); nadia.zine@univ-lyon1.fr (N.Z.); \\ abdelhamid.errachid-el-salhi@univ-lyon1.fr (A.E.) \\ 2 Bacelona Microelectronics Institute IMB-CNM (CSIC), 08193 Bellaterra, Spain; \\ albert.alcacer@imb-cnm.csic.es (A.A.); angelos.streklas@imb-cnm.csic.es (A.S.); \\ joan.bausells@imb-cnm.csic.es (J.B.) \\ 3 Institut de Ciència de Materials de Barcelona (CSIC), Campus de la U.A.B, 08193 Bellaterra, Spain; \\ teixidor@icmab.es \\ * Correspondence: juan.gallardo-gonzalez@univ-lyon1.fr; Tel.: +33-763-165-664 \\ + Presented at the Eurosensors 2017 Conference, Paris, France, 3-6 September 2017.
}

Published: 7 August 2017

\begin{abstract}
In the present work a highly sensitive ion-selective microelectrode for the detection of amphetamine is presented. For this purpose, a novel ion-par complex based on the metallocarborane, cobalt bis(dicarbollide) anion $\left(\left[3,3^{\prime}-\mathrm{Co}\left(1,2-\mathrm{C}_{2} \mathrm{~B}_{9} \mathrm{H}_{11}\right)_{2}\right]^{-}\right)$coupled to amphetamonium cation has been prepared as the active site for amphetamine recognition. The prepared ion-par complex was incorporated to a PVC-type sensitive membrane. It was then drop-casted on the top of a gold microelectrode previously modified with a solid contact layer of polypyrrole. This novel amphetamine microsensor has provided excellent and quick response within the range $10^{-5} \mathrm{M}$ to $10^{-3} \mathrm{M}$ of amphetamine concentration, a limit of detection of $12 \mu \mathrm{M}$ and a slope of $60.1 \mathrm{mV} /$ decade. It was also found to be highly selective toward some potential interference compounds when compared to amphetamine.
\end{abstract}

Keywords: amphetamine; ion-par complex; metallocarboranes

\section{Introduction}

Nowadays, illicit drugs are considered one of the major concerns for developed countries due to the potential of bringing about all types of healthy problems. Although cannabis and cocaine are by far the most used narcotic, amphetamine and methylenedioxy-methamphetamine (MDMA) are becoming more and more popular. The last rapport from the European Monitoring Centre for Drug and Drug Consumption (MCDDC) estimated that about 1.3 and 2.1 million of person have consumed amphetamine and MDMA respectively in the last year [1]. Nevertheless, not only the healthy problems related to drug use are a matter of concern but also the black economy that it implies has become alarming. The estimated annual value of the retail market for amphetamine in Europe is about EUR 1.8 billion according to the MCDDC what means that 76 tonnes of amphetamine were consumed in the last year [2]. Collecting information concerning illicit drug uses plays a vital role to help low enforcement agencies in prevention and fight against criminal organizations. At present, chemical analysis are widely used to complement the epidemiology 
studies of population traditionally carried out in surveys. Particularly, the chemical analysis of wastewater is a powerful tool to monitor the pattern and trends of illicit drugs consumption in a community [3,4]. However, as in the cases of amphetamine and its derivatives most analytical techniques used to analyze wastewater samples and detect the illicit compounds are based on ex-situ studies like colorimetric measurements, capillary electrophoresis, HPLC or GC-MS among others. Recently, researchers from our group have presented an all-solid-state amphetamine-selective electrodes based on the crown ether dibenzo-18-crown- 6 as amphetamine ionophore [5] and K. Graniczkowska et al. presented a capacitive sensor able to detect trace amounts of a precursor of the amphetamine synthesis [6]. The aim of this work is to present a highly selective microsensor easy to handle for in situ amphetamine detection by fasts potentiometric measurements at real time in both soft (street samples) and harsh environment (wastewater). To accomplish this, a novel amphetamine ion-par complex has been prepared as the active component of a polymeric sensitive membrane for amphetamine recognition. Metallocarboranes sandwich anions of the type $\left[3,3^{\prime}-\mathrm{Co}\left(1,2-\mathrm{C}_{2} \mathrm{~B}_{9} \mathrm{H}_{11}\right)_{2}\right]^{-}$have been reported for analysis applications due to their very good chemical properties (stability, hydrophobicity and extreme organophility) when isolated with organic bases of the type [cation- $\mathrm{NH}]^{\mathrm{n}+} \mathrm{n}\left[3,3^{\prime}-\mathrm{Co}\left(1,2-\mathrm{C}_{2} \mathrm{~B}_{9} \mathrm{H}_{11}\right)_{2}\right]^{-}$[7]. This strategy allows incorporating the target molecule to the sensitive membrane enhancing the sensor's performance and the selectivity. The ion-par complex $\left[\mathrm{C}_{9} \mathrm{H}_{13} \mathrm{NH}\right]^{+}\left[3,3^{\prime}-\mathrm{Co}\left(1,2-\text { closo }-\mathrm{C}_{2} \mathrm{~B}_{9} \mathrm{H}_{11}\right)_{2}\right]^{-}$has been synthetized and the product obtained has been characterized by FTIR.

\section{Materials and Methods}

\subsection{Preparation of $\left[\mathrm{C}_{9} \mathrm{H}_{13} \mathrm{NH}\right]^{+}\left[3,3^{\prime}-\mathrm{Co}\left(1,2-\text { closo }-\mathrm{C}_{2} \mathrm{Bg}_{9} \mathrm{H}_{11}\right)_{2}\right]^{-}$}

The ion-par complex $\left[\mathrm{C}_{9} \mathrm{H}_{13} \mathrm{NH}\right]^{+}\left[3,3^{\prime}-\mathrm{Co}\left(1,2-\text { - closo- } \mathrm{C}_{2} \mathrm{~B}_{9} \mathrm{H}_{11}\right)_{2}\right]^{-}$has been obtained by ion-exchange procedure. An acidic solution of $\mathrm{Cs}\left[3,3^{\prime}-\mathrm{Co}\left(1,2-\text { closo }^{-}-\mathrm{C}_{2} \mathrm{~B}_{9} \mathrm{H}_{11}\right)_{2}\right]$ was extracted with diethyl ether to obtain the metallocarborane in the form $\mathrm{H}\left[3,3^{\prime}-\mathrm{Co}\left(1,2-\text { closo }-\mathrm{C}_{2} \mathrm{~B}{ }_{9} \mathrm{H}_{11}\right)_{2}\right]$. Then, the diethyl ether was evaporated under vacum and the residue was diluted with water to generate $0.05 \mathrm{M}$ solution of $\mathrm{H}\left[3,3^{\prime}-\mathrm{Co}\left(1,2-\text { closo }_{2}-\mathrm{C}_{2} \mathrm{~B}_{9} \mathrm{H}_{11}\right)_{2}\right]$ (solution 1). Amphetamine was dissolved in water and with the minimum quantity of $\mathrm{HCl}$ to prepare $0.05 \mathrm{M}$ acidic solution (solution 2). Next, $20 \mathrm{~mL}$ of solution 1 and $20 \mathrm{~mL}$ of solution 2 were mixed and after stirring a yellow precipitate was obtained. This was filtered off, washed with $\mathrm{HCl} 0.1 \mathrm{M}$ and dried in vacuum.

\subsection{Sensitive Membrane}

The polymeric membrane selective to amphetamine was made of $31 \mathrm{wt} \%$. PVC as the polymeric matrix, $65 \mathrm{wt} \%$. dibutyl phthalate as plasticizer and 4 wt $\%$ of $\left[\mathrm{C}_{9} \mathrm{H}_{13} \mathrm{NH}\right]^{+}\left[3,3^{\prime}-\mathrm{Co}\left(1,2-\text { closo- } \mathrm{C}_{2} \mathrm{~B}_{9} \mathrm{H}_{11}\right)_{2}\right]^{-}$ion-par complex. All components were dissolved in THF. The solution mixture was drop-casted onto the gold microelectrode already modified with the polypyrrole conductive layer and let the solvent evaporate overnight.

\section{Results and Discussion}

\subsection{The Ion Par Complex $\left[\mathrm{C}_{9} \mathrm{H}_{13} \mathrm{NH}\right]^{+}\left[3,3^{\prime}-\mathrm{Co}\left(1,2-\text { closo- } \mathrm{C}_{2} \mathrm{~B}_{9} \mathrm{H}_{11}\right)_{2}\right]^{-}$}

The electroactive ion-par complex obtained was characterized by FT-IR spectroscopy. The IR spectrum shows evidence of hydrogen or dihydrogen bonding at the B-H and $\mathrm{Cc}-\mathrm{H}$ or $\mathrm{ArC}-\mathrm{H}$ stretching regions. The IR spectrum can be presented under request.

\subsection{Sensor's Respons Characteristic: Potentiometric Measurements}

The performance of the amphetamine-selective microsensor was determined following the generalized standard addition method [8] by titration of amphetamine sulfate solutions from $10^{-7}$ to $10^{-3} \mathrm{M}$. Potentiometric measurements provided information regarding the Nernstian behavior, the limit of detection and the response time. Results are summarized in Table 1. 
Table 1. Characteristic response of the amphetamine selective microsensor.

\begin{tabular}{cc}
\hline Parameter & Amphetamine Selective Microsensor \\
\hline Slope (mV/decade) & 60.1 \\
Limit of detection $(\mathrm{M})$ & $1.2 \times 10^{-5}$ \\
Time of response (s) & $<10$ \\
\hline
\end{tabular}

As it can be observed in Figure 1, the device developed integrates an array of four gold working microelectrodes, two $\mathrm{Ag} / \mathrm{AgCl}$ pseudo-reference microelectrodes and one gold auxiliary microelectrode. So, four sensors can measure at the same time using a single miniaturized device. It is very useful not only for multi-detection applications but also to obtain fast statistical values. As reported in Table 1 the amphetamine-selective microsensor presents a Nernstian slope of $60.1 \mathrm{mV} /$ decade of amphetamine concentration. This is near to the theoretical value of $59 \mathrm{mV} / \mathrm{dec}$ de expected for a monovalent cation such as amphetamine in the protonated form. The limit of detection was extracted as the crossing point of the two linear segments from the calibration curve shown in Figure 1. The microsensor presents a limit of detection of $12 \mu \mathrm{M}$. The time needed by the sensor to achieve a stable response was found to be less than $10 \mathrm{~s}$.

a)

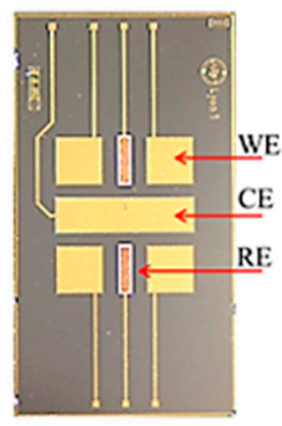

b)

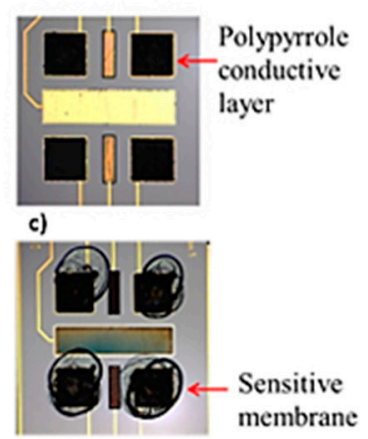

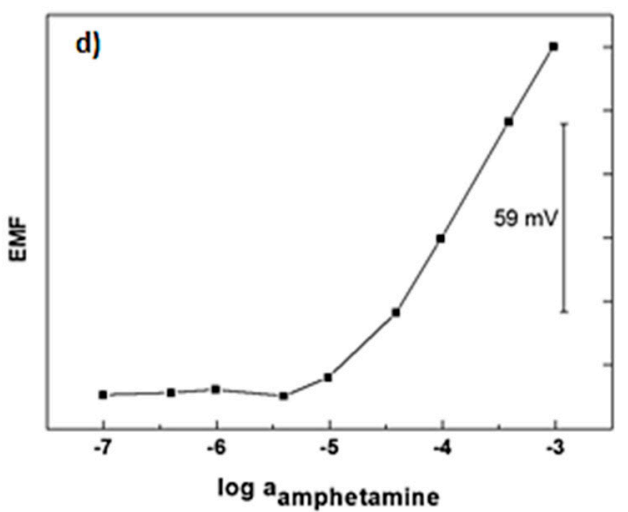

Figure 1. (a) transducer holding an array of four bare-gold working microelectrodes (WE), one bare-gold counter microelectrode (CE) and two $\mathrm{Ag} / \mathrm{AgCl}$ reference microelectrodes (RE); (b) WEs electrochemically modified with polypyrrole solid conductive layer; (c) the polymeric membrane including the ion-par complex $\left[\mathrm{C}_{9} \mathrm{H}_{13} \mathrm{NH}\right]^{+}\left[3,3^{\prime}-\mathrm{Co}\left(1,2-\text { closo- } \mathrm{C}_{2} \mathrm{~B}_{9} \mathrm{H}_{11}\right)_{2}\right]^{-}$drop casted on the top of WEs; (d) Potentiometric calibration curve of the amphetamine selective microelectrode developed.

\subsection{Selectivity}

Coefficients of selectivity, $K_{I J}{ }^{\text {Pot }}$, were obtained following the fixed interference method [9]. A titration of amphetamine sulfate standard solutions was carried out from $10^{-7}$ to $10^{-3} \mathrm{M}$ in $25 \mathrm{~mL}$ of $1 \mathrm{mM}$ solution of $\mathrm{N}$-formyl amphetamine, methylbenzylamine or phenylalanine respectively. Results are summarized in Table 2.

Table 2. Selectivity coefficient of the amphetamine-selective microsensor.

\begin{tabular}{cc}
\hline Interference & $K_{I J}^{\text {Pot }}$ \\
\hline N-formyl amphetamine & -2.15 \\
Methylbenzylamine & -2.09 \\
Phenylalanine & -2.09 \\
\hline
\end{tabular}

The results obtained confirm the high selectivity of the amphetamine-selective microsensor. The $K_{I I}{ }^{\text {Pot }}$ values show that the response toward amphetamine in the presence of these amino/amide compounds structurally similar is around hundred times more sensitive. It is well known that plasticizers play an important role concerning the lifetime, sensitivity and selectivity of ion selective electrodes. However, introducing the target molecule into the polymeric membrane through the 
ion-par complex $\left[\mathrm{C}_{9} \mathrm{H}_{13} \mathrm{NH}\right]^{+}\left[3,3^{\prime}-\mathrm{Co}\left(1,2-\text { closo }-\mathrm{C}_{2} \mathrm{~B}_{9} \mathrm{H}_{11}\right)_{2}\right]^{-}$synthetized is crucial to achieve those values of selectivity.

\section{Conclusions}

The development of an all-solid-state and highly selective amphetamine micro sensors has been reported. The ion par complex $\left[\mathrm{C}_{9} \mathrm{H}_{13} \mathrm{NH}\right]^{+}\left[3,3^{\prime}-\mathrm{Co}\left(1,2-\text { closo }-\mathrm{C}_{2} \mathrm{~B}_{9} \mathrm{H}_{11}\right)_{2}\right]^{-}$has been synthetized as the active part of a PVC-type polymeric membrane for amphetamine recognition. The microsensor showed Nernstian response with a slope of $60.1 \mathrm{mV} /$ decade within the concentration range $10^{-5} \mathrm{M}$ to $10^{-3} \mathrm{M}$ of amphetamine, limit of detection of $12 \mu \mathrm{M}$ and a time of response less than $10 \mathrm{~s}$. The cross-selectivity study in the presence of $\mathrm{N}$-formyl amphetamine, methylbenzylamine and phenylalanine shows that the sensor's response toward amphetamine is highly selective. In addition, the miniaturized device developed is able to produce reliable and real-time data using four independent amphetamine-selective microsensors at the same time.

Acknowledgments: The authors acknowledge the financial support from the European Union's Horizon 2020 research and innovation programme entitled MicroMole and HEARTEN grant agreement No. 653626 and No. 643694 respectively

Author Contributions: Authors made substantial contributions to conception, design, and/or acquisition of data, and/or analysis and interpretation of data. Authors have also participated in drafting the article or revising it critically for important intellectual content and have given final approval of the version to be submitted and any revised version.

Conflicts of Interest: The authors do not have any conflict of interest to be declared.

\section{References}

1. European Monitoring Centre for Drugs and Drug Addiction. European Drug Report 2016: Trends and Developments; Publication Office of the European Union: Luxembourg, 2016.

2. European Monitoring Centre for Drugs and Drug Addiction and Europol. EU Drug Markets Report. In-depth Analysis; EMCDDA-Europol Joint Publications, Publications Office of the European Union: Luxembourg, 2016.

3. European Monitoring Centre for Drugs and Drug Addiction. Assesing Ilicit Drugs in Wastewater: Advances in Wastwater-Based Drug Epidemiology; Publication Office of the European Union: Luxembourg, 2016.

4. Castiglioni, S.; Thomas, K.V.; Kasprzyk-Hordern, B.; Vandam, L.; Griffiths, P. Testing wastewater to detect illicit drugs: State of the art, potential and research needs. Sci. Total Environ. 2014, 487, 613-620.

5. Gallardo-Gonzalez, J.; Baraket, A.; Bonhomme, A.; Zine, N.; Sigaud, M.; Bausells, J.; Errachid, A. Sensitive Potentiometric Determination of Amphetamine with a Micro Ion-Selective Electrode. Anal. Lett. 2017, doi:10.1080/00032719.2017.1326053.

6. Graniczkowska, K.; Pütz, M.; Hauser, F.M.; de Saeger, S.; Beloglazova, N.V. Capacitive sensing of $\mathrm{N}$-formylamphetamine based on immobilized molecular imprinted polymers. Biosens. Bioelectron. 2016, 92, 741-747.

7. Plesek, J.; Base, K.; Mares, F.; Hanousek, F.; Hermanek, S. Potential Uses Of Metallocarborane Sadnwich Anions For Analysis, Characterization And Isolation Of Various Cations and Organic Bases. Collect. Czech. Chem. Commun. 1983, 49, 2776-2789.

8. Saxberg, B.E.H.; Kowalski, B.R. Generalized standard addition method. Anal. Chem. 1979, 51, 1031-1038.

9. Bakker, E.; Pretsch, E.; Bühlmann, P. Selectivity of Potentiometric Ion Sensors. Anal. Chem. 2000, 72, $1127-1133$.

(C) 2017 by the authors. Licensee MDPI, Basel, Switzerland. This article is an open access article distributed under the terms and conditions of the Creative Commons Attribution (CC BY) license (http://creativecommons.org/licenses/by/4.0/). 